\title{
Image Sequence Segmentation Combining Global Labeling and Local Relabeling and its Application to Materials Science Images
}

\author{
Jarrell W. Waggoner ${ }^{a}$, Jeff Simmons ${ }^{b}$, and Song Wang ${ }^{a}$ \\ ${ }^{a}$ University of South Carolina, Columbia, SC 29208, USA; \\ ${ }^{b}$ Materials and Manufacturing Directorate, Air Force Research Labs, Dayton, OH 45433, USA
}

\begin{abstract}
Accurately segmenting a series of 2D serial-sectioned images for multiple, contiguous 3D structures has important applications in medical image processing, video sequence analysis, and materials science image segmentation. While 2D structure topology is largely consistent across consecutive serial sections, it may vary locally because a $3 \mathrm{D}$ structure of interest may not span the entire $2 \mathrm{D}$ sequence. In this paper, we develop a new approach to address this challenging problem by considering both the global consistency and possible local inconsistency of the $2 \mathrm{D}$ structural topology. In this approach, we repeatedly propagate a 2D segmentation from one slice to another, and we formulate each step of this propagation as an optimal labeling problem that can be efficiently solved using the graph-cut algorithm. Specifically, we divide the optimal labeling into two steps: a global labeling that enforces topology consistency, and a local labeling that identifies possible topology inconsistency. We justify the effectiveness of the proposed approach by using it to segment a sequence of serial-section microscopic images of an alloy widely used in material sciences and compare its performance against several existing image segmentation methods.
\end{abstract}

Keywords: Segmentation, Materials, Propagation, Topology Constraints, Local and Global

\section{INTRODUCTION}

Images of 3D structures made up of multiple 2D slices play an important role in a myriad of fields, including video analysis and compression, ${ }^{1}$ medical imaging, ${ }^{2}$ and civil and industrial materials science. ${ }^{3}$ Everything from tomographic sequences and 3D structure volumes to medical CT/MRI and video sequences make up this vast array of serial-sectioned data, which form a collectively challenging set of problems for image segmentation. While significant research has been made on many of these problems, one basic issue has not been specifically and systematically addressed: how to model and identify both 2D topology consistency and possible inconsistency across slices in image sequence segmentation. In this paper, we address this issue by developing an image sequence segmentation method, which propagates a 2D segmentation sequentially from one slice to another.

As illustrated in Fig. 1, the 3D structure of interest is made up of multiple contiguous substructures, and the 2 D topology ${ }^{4,5}$ consistency is reflected by the fact that nearby series sections show similar substructures (e.g., $\left.s_{1} \leftrightarrow s_{2}\right)$. However, inconsistency may be introduced when the series section moves into a new substructure or moves out of an existing substructure (e.g., $s_{2} \leftrightarrow s_{3}$, and $s_{3} \leftrightarrow s_{4}$ ). The segmentation of such structures becomes a very challenging problem when the number of substructures is large and such 2D topology changes are not known. This is a common phenomenon in many fields, such as segmenting cells in medical imaging, grain structures in materials science, and crowd scenes in video surveillance. Some previous work employs direct $3 \mathrm{D}$ segmentation instead of segmenting 2D slices sequentially; however, direct 3D segmentation may not work when there is large intensity and contrast changes across these slices ${ }^{6}$ and/or inter-slice resolution is much lower than the intra-slice resolution. ${ }^{7}$ The method proposed in this paper specificially focuses on segmenting images with a large number of contiguous substructures.

Further author information: (Send correspondence to J.W.W.)

J.W.W.: E-mail: waggonej@email.sc.edu, Telephone: 847-261-4747

J.S.: E-mail: jeff.simmons@wpafb.af.mil

S.W.: E-mail: songwang@cec.sc.edu, Telephone: 803-777-2487

Computational Imaging $X$, edited by Charles A. Bouman, llya Pollak,

Patrick J. Wolfe, Proc. of SPIE-IS\&T Electronic Imaging, SPIE Vol. 8296, 829606

(c) 2012 SPIE-IS\&T · CCC code: 0277-786X/12/\$18 - doi: 10.1117/12.906471 

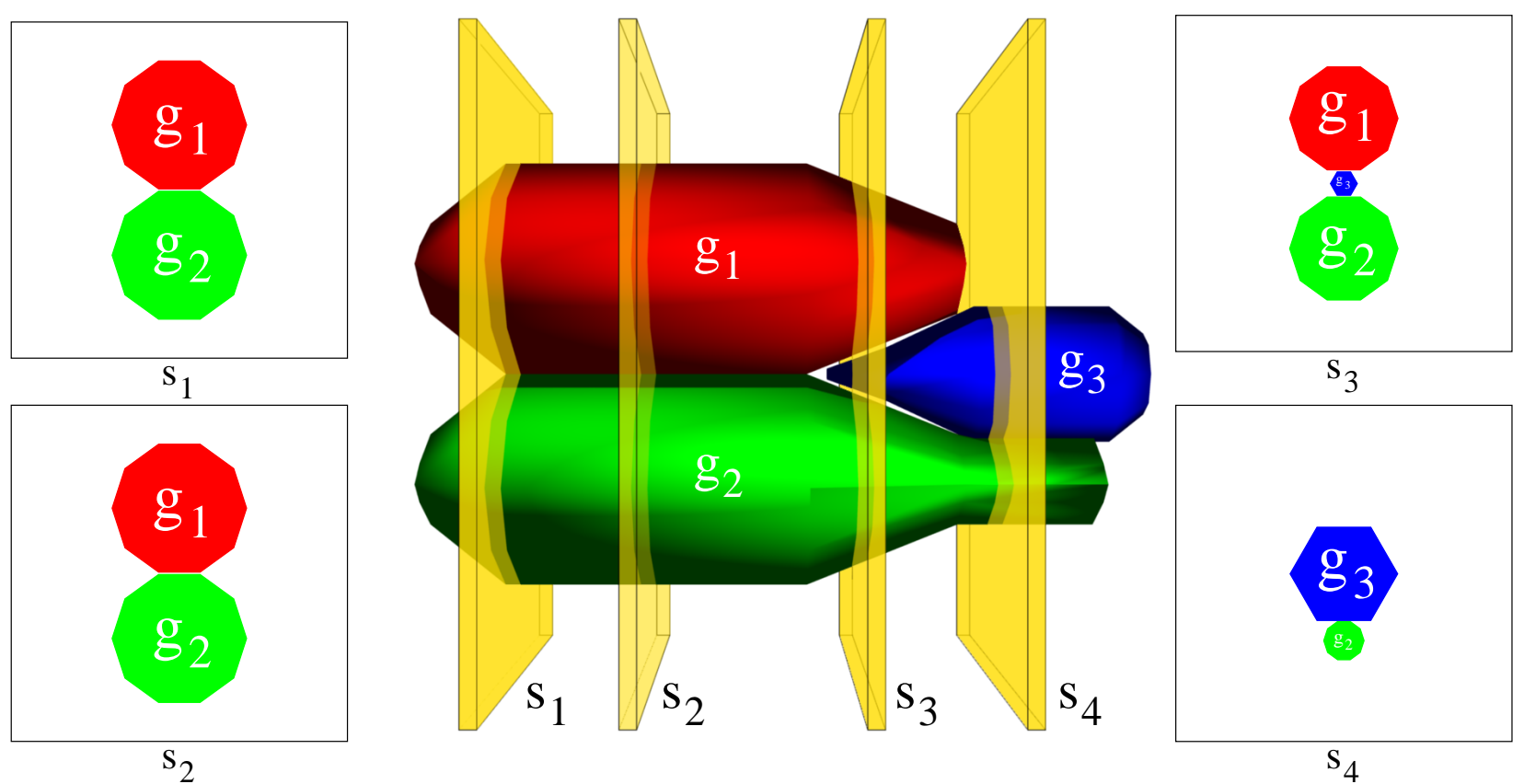

Figure 1: An illustration of 2D structure topology consistency $\left(s_{1} \leftrightarrow s_{2}\right)$ and possible inconsistency $\left(s_{2} \leftrightarrow s_{3}\right.$ and $\left.s_{3} \leftrightarrow s_{4}\right)$ across slices.

Related to the proposed method are tracking and tracking-based segmentation methods that have been successfully used in video ${ }^{8}$ and medical imaging. ${ }^{9}$ However, they typically track a single structure, or a small number of substructures, and they cannot usually well-handle topology inconsistency and large numbers of substructures. The segmentation of a large number of substructures is usually obtained by a $2 \mathrm{D}$ segmentation algorithm such as watershed ${ }^{10-12}$ and normalized cut. ${ }^{8,13,14}$ For some of these 2D segmentation methods, such as watershed, 2D topology consistency can be imposed across slices to achieve more consistent image sequence segmentation. ${ }^{15}$ In Section 5, we conduct experiments to compare the performance of the proposed method with the normalized cut and watershed methods.

In this paper, we develop a new method to segment a sequence of images by repeatedly propagating a $2 \mathrm{D}$ segmentation from one slice to another. We formulate this process as an optimal labeling that can be efficiently solved using the graph-cut algorithm. To maintain general 2D topology consistency across slices, we first run a global labeling to produce an initial segmentation on the new slice. We then run a series of local relabelings to refine the segmentation by identifying and correcting possible 2D topology inconsistencies.

\subsection{Application to Materials Science Images}

In this paper, we apply the proposed method to segment metallic materials science images. For simplicity, we also use material science images for our algorithm development. With many desirable mechanical, electrical, thermal, chemical, and manufacturing properties, metallic materials play an important role in both civil and military industries. These properties are strongly dependent on the detailed microscopic substructure of the material. ${ }^{16}$ In particular, most metallic materials consist of a large number of microscopic crystals, or "grains," and accurate segmentation of these grains can substantially facilitate the analysis of material properties and shorten the design period for new metallic materials.

Automatic segmentation of metallic images is a highly challenging problem. Aside from the possible topology inconsistency across slices and the vast number of substructures present, these images also contain various kinds of noise and ambiguities, as shown by the two serial-section images of a titanium sample in Fig. 2 (a) and (b), where some grain boundaries may be less distinct than other boundaries (e.g., $\left.g_{1}\right)$. In addition, some undesired scratches inside the grains may exhibit high intensity similar to grain boundaries (e.g., $g_{2}$ ). Such image noise and ambiguity 


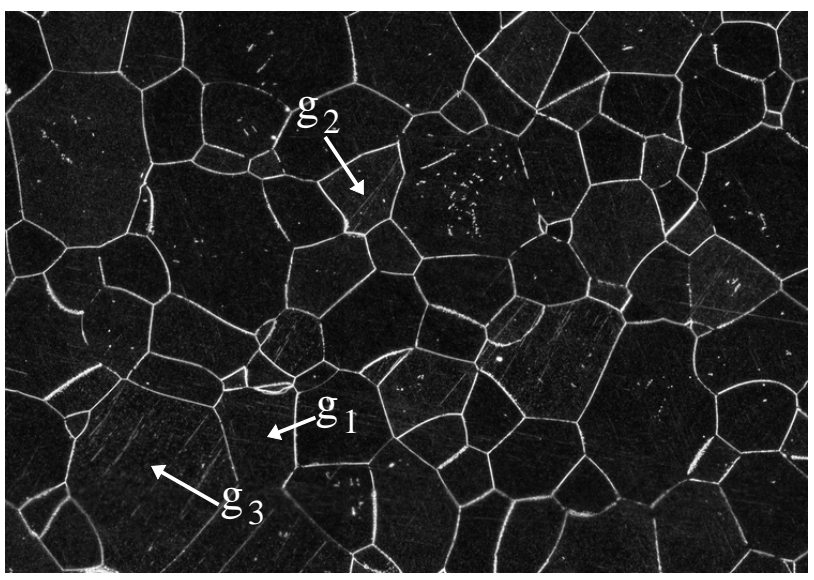

(a)

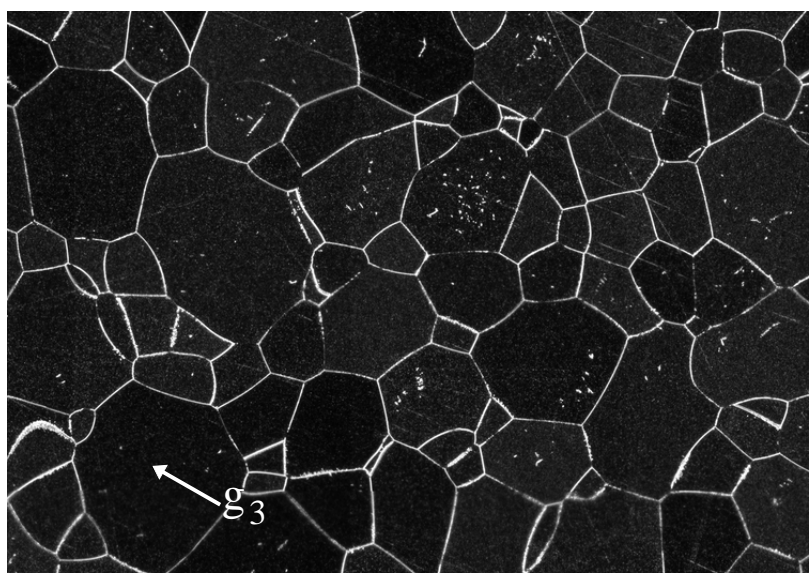

(b)

Figure 2: An illustration of the challenges in metallic image segmentation. (a) \& (b) Two image slices of a titanium sample. Grain $g_{1}$ contains indistinct boundary segments, grain $g_{2}$ contains undesired image noise, and grain $g_{3}$ exhibits inconsistent intensity between these two slices.

makes it difficult to accurately extract all the grain boundaries by edge detection ${ }^{17,18}$ or intensity thresholding. ${ }^{19}$ Furthermore, the chemical processing, together with lighting changes during the microscopic imaging, often leads to inconsistent image intensity across slices. For example, grain $g_{3}$ exhibits different intensities between the two slices shown in Fig. 2. This makes it difficult to directly apply a 3D volumetric image segmentation method. ${ }^{20}$ We believe the capability of the proposed method to handle such a challenging problem is a good indication that it can be extended to other image sequence segmentation applications.

\section{OPTIMAL LABELING WITH GRAPH CUT}

In this paper, we primarily use the multi-labeling framework described in, ${ }^{21-23}$ where image segmentation is formulated as an assignment of labels to each pixel and the pixels with the same label constitute a segment. As described in, ${ }^{21-23}$ the objective of the multi-labeling algorithm is to find a labeling function $f$ by minimizing the energy function

$$
E(f)=\sum_{p \in \mathcal{P}} D_{p}\left(f_{p}\right)+\sum_{\{p, q\} \in \mathcal{N}} V_{p q}\left(f_{p}, f_{q}\right),
$$

where $\mathcal{P}$ is the set of image pixels, $f_{p}$ is the label of pixel $p \in \mathcal{P}$, and $\mathcal{N}$ is the set of pixel pairs that are neighbors. In this paper, a pair of pixels are considered neighbors if they are 4-connected. The data term $D_{p}\left(f_{p}\right)$ describes the cost of assigning label $f_{p}$ to pixel $p$ and the smoothness term $V_{p q}\left(f_{p}, f_{q}\right)$ describes the cost of assigning labels $f_{p}$ and $f_{q}$ to two neighboring pixels $p$ and $q$, respectively. In, ${ }^{24}$ it is shown that finding the globally optimal labeling that minimizes energy Eq. (1) is an NP-hard problem. However, the minimum graph-cut algorithm can be applied to efficiently find a locally optimal labeling. For our purposes, we will define a specialized data term $D_{p}\left(f_{p}\right)$ and smoothness term $V_{p q}\left(f_{p}, f_{q}\right)$ for propagating a $2 \mathrm{D}$ image segmentation from one slice to another.

\section{GLOBAL LABELING}

Fundamentally, the proposed algorithm repeatedly propagates 2D image segmentations from one slice to another. The 2D segmentation of an initial slice is constructed either manually or using an automatic or semiautomatic method. We first focus on the entire image by enforcing consistent topology during this propagation. We use the multilabeling algorithm described in Section 2 to achieve this purpose.

Let's consider propagating the 2D segmentation from slice $S$ to a neighboring slice $I$. The segmentation of slice $S$ is available in the form of a label matrix, where each pixel is assigned a label. In this paper, we use $\left\{g_{1}, g_{2}, \ldots, g_{n}\right\}$ to identify labels and segments when there is no ambiguity; $n$ is the total number of segments in slice $S$. Our goal is then to find an optimal labeling on slice $I$, using the same set of labels in $S$, such that the 
identified 2D segments with the same label in these two slices represent two different serial sections of the same $3 \mathrm{D}$ structure.

Considering the structural continuity, we first make the assumption that the serial sections of the same structure in slices $S$ and $I$ have good spatial overlap, while their 2D boundaries may show limited rigid or nonrigid deformation. This is a valid assumption in a number of fields (e.g., medical and materials) and is valid in a number of special cases in many other fields (e.g., high-speed video). With this assumption, we use the following two steps to define the data term $D_{p}$ for labeling slice $I$ :

1. Morphologically dilate each segment in slice $S$, as illustrated in Fig. 3 (a).

2. Map the dilation results onto slice $I$. We set $D_{p}\left(g_{i}\right)=0$ if pixel $p$ is located in the dilated segment of $g_{i}$, and $D_{p}\left(g_{i}\right)=\infty$ otherwise. For example, Fig. $3(\mathrm{c})$ is the data term defined for three pixels $p_{1}, p_{2}$, and $p_{3}$ shown in Fig. $3(\mathrm{~b})$.

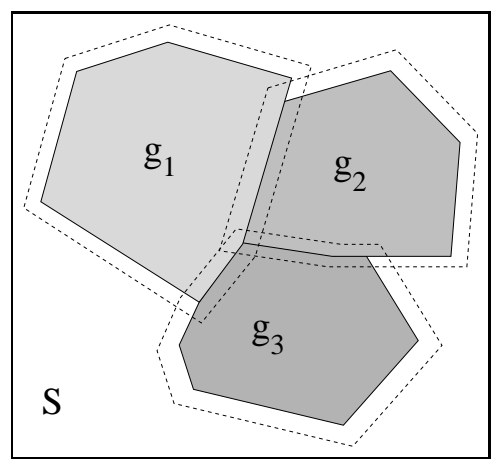

(a)

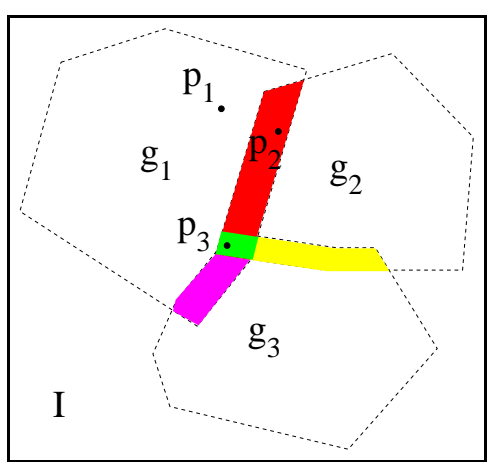

(b)

\begin{tabular}{c|c|c|c}
$D$ & $g_{1}$ & $g_{2}$ & $g_{3}$ \\
\hline$p_{1}$ & 0 & $\infty$ & $\infty$ \\
$p_{2}$ & 0 & 0 & $\infty$ \\
$p_{3}$ & 0 & 0 & 0
\end{tabular}

(c)

Figure 3: An illustration of defining data term $D_{p}$ in global labeling. (a) Dilate $g_{1}, g_{2}$, and $g_{3}$ in slice $S$. (b) Map dilated segments to slice $I$. (c) Data term $D_{p}$ defined for pixels $p_{1}$ to $p_{3}$ in (b).

Clearly, this definition of the data term enforces the structural continuity between two neighboring slices. The dilation size is determined by the maximum possible spatial variation between the $2 \mathrm{D}$ serial sections of the same 3D structure from one slice to another. In general, the smaller the distance between two neighboring slices, the smaller the dilation size needed. Thus, though it is a free parameter, the dilation size can be computed directly from knowledge of the application domain and/or imaging process in many instances. In this paper, we select a constant dilation size, derived from properties of the metallic material slices we segment, for all our experiments.

To preserve consistent topology between two neighboring slices $S$ and $I$, we further define the smoothness term $V_{p q}$ as

$$
V_{p q}\left(f_{p}, f_{q}\right)=\left\{\begin{array}{lc}
0, & f_{p}=f_{q} \\
\infty, & f_{p} \neq f_{q} \wedge f_{p} \Leftrightarrow f_{q} \\
\frac{255}{\max \left(I_{p}, I_{q}\right)}, & f_{p} \neq f_{q} \wedge f_{p} \Leftrightarrow f_{q}
\end{array}\right.
$$

where $f_{p} \Leftrightarrow f_{q}$ indicates that the segments with label $f_{p}$ and the segment with label $f_{q}$ are neighboring segments in slice $S$. When $f_{p} \nRightarrow f_{q}$, we set the smoothness term to be $\infty$ to prevent $f_{p}$ and $f_{q}$ from being neighbors when labeling the new slice $I . I_{p}$ and $I_{q}$ are the pixel intensities (in the range of $[0,255]$ for the images we use in this paper) at $p$ and $q$ in slice $I$. We define the smoothness term to be $\frac{255}{\max \left(I_{p}, I_{q}\right)}$ for the case $f_{p} \Leftrightarrow f_{q}$ to penalize boundaries that pass through low-intensity pixels. As illustrated in Fig. 2, the low intensity pixels are more likely part of the grain interior for the example metallic slices.

With the data term and the smoothness term, we can apply the graph-cut algorithm to obtain an optimal labeling on slice $I$. Fig. 4 shows an example of global labeling on a metallic image. For clarity, we show a magnified view of the labeling result in a small window. 


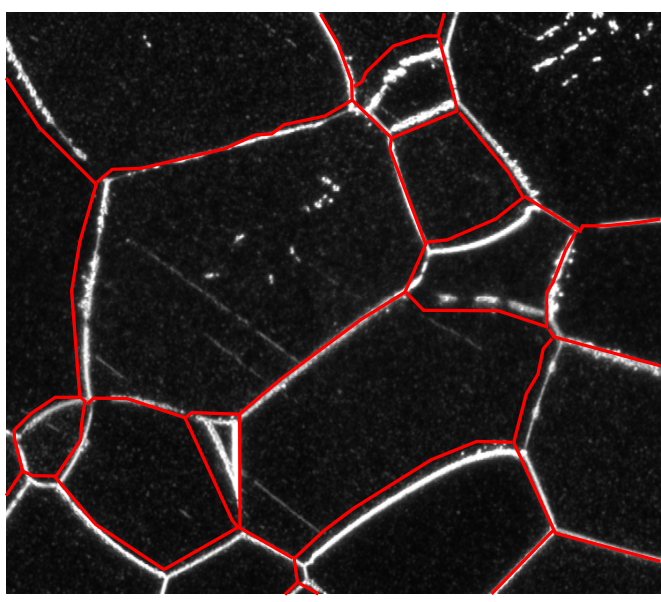

(a)

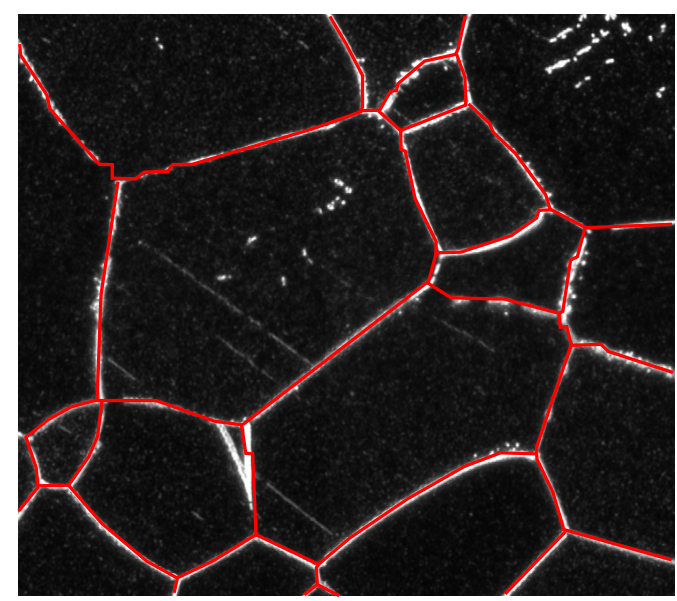

(b)

Figure 4: Magnified view of the global labeling in a small window. (a) Grain boundaries in slice $S$ copied to slice $I$. (b) Grain boundaries in slice $I$ after global labeling.

\section{LOCAL RELABELING}

The global labeling discussed in Section 3 is all that is necessary to propagate a segmentation from one slice to a neighboring slice while preserving the pairwise neighboring relations between segments, which we call topology in this paper. However, there are three problems that are either introduced by the global labeling or are not addressed by global labeling. Note that we adopt materials science-specific terminology (e.g., "grains" instead of "segments") to make this discussion easier, though the ideas presented here can be adapted and generalized to any of a number of domains.

Problem I: Certain grains in slice $S$ may disappear in slice $I$ when the serial section plane moves out of these grains, as shown by grain $g_{2}$ in Fig. 5 . Such grains are usually very small and completely covered by the dilation of its neighboring grains in slice $S$. The global labeling may remove such grains in slice $I$ automatically, if the removal of such grains does not change the pairwise neighboring relations of other grains, as shown in Fig. 5 (d-e). However, in some cases, the disappearance of such grains may change the pairwise neighboring relations of other grains. An example is shown in Fig. 5, where the disappearance of $g_{2}$ makes $g_{1}$ and $g_{3}$ neighbors, which leads to an infinity smoothness term according to Eq. (2). As a result, the global labeling is undesirably forced to retain the label $g_{2}$ as shown in Fig. 5 (b).

Problem II: Grains with small size in the new slice $I$ may be removed by the global labeling if its absence does not change the pairwise neighboring relations in other grains. An example is shown in Fig. 6, where the global labeling in slice $I$ does not retain label $g_{4}$, causing it to be merged into segment $g_{2}$. The reasons are threefold: (i) the dilations of $g_{1}, g_{2}$, and $g_{3}$ in slice $S$, when mapped to slice $I$, cover grain $g_{4}$ entirely. (ii) The absence of $g_{4}$ in $I$ does not alter the pairwise neighboring relations of other grains defined in $S$ (i.e., $g_{1}, g_{2}$, and $g_{3}$ are neighbors, whether $g_{4}$ is preserved or removed). This way, the removal of $g_{4}$ does not introduce infinity penalty in the smoothness term Eq. (2). (iii) The energy function Eq. (1) favors the usage of fewer labels: the fewer the labels, the shorter the total boundaries, and the smaller the energy.

Problem III: A new grain may appear when moving from slice $S$ to slice $I$, as shown by the grain $g_{4}$ in Fig. 7 (c). As shown in Fig. 7 (b), the global labeling cannot introduce a new label for this new grain in $S$. There are two cases for this problem. Case I: the appearance of this new grain does not change the pairwise neighboring relations of other grains defined in slice $S$, as shown by the example in Fig. 7 (a-c). Case II: the appearance of this new grain does change the pairwise neighboring relations of other grains defined in slice $S$, as shown by the example in Fig. 7 (d-f).

In this section, we propose three strategies to address the above problems. In these strategies, we identify local regions with such problems, modify the data term and/or smoothness term, and then run the optimal labeling algorithm to relabel the grains in such local regions. 


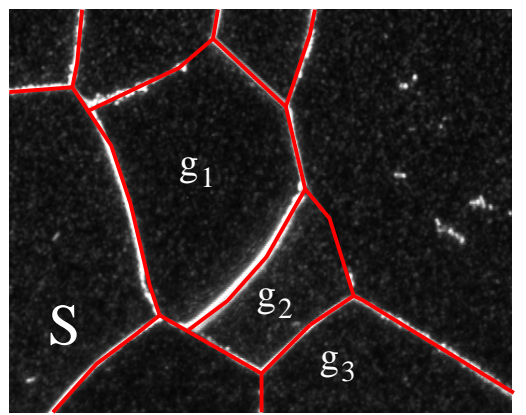

(a)

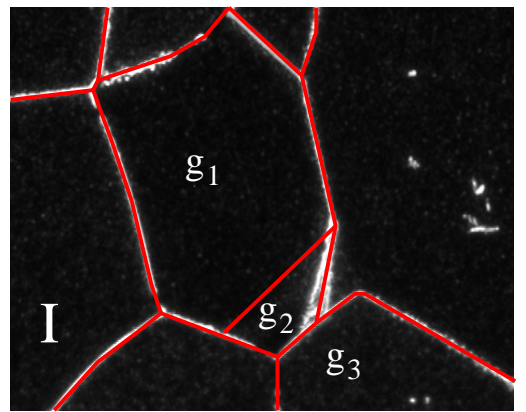

(b)

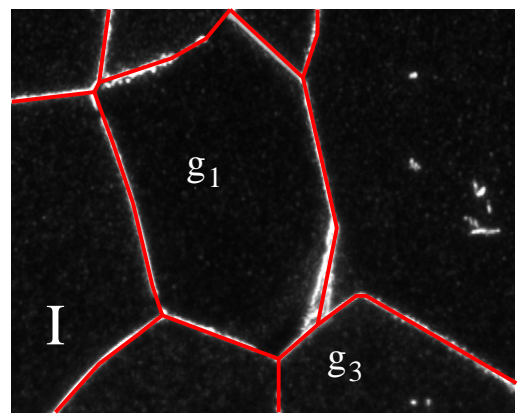

(c)

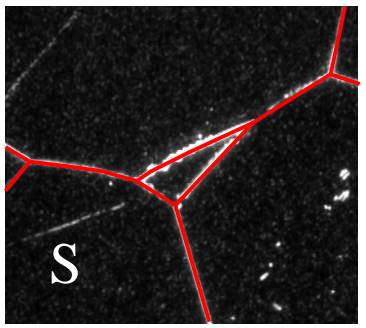

(d)

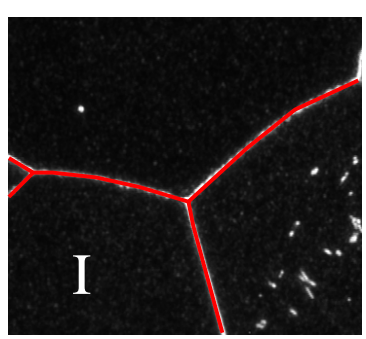

(e)

Figure 5: An illustration of Problem I. (a) Slice $S$, where grains $g_{1}$ and $g_{3}$ are not neighbors. (b) Slice $I$, where the global labeling refuses to remove $g_{2}$, because it would cause $g_{1}$ and $g_{3}$ to be neighbors. (c) The local relabeling result on slice $I$ after applying Strategy I. (d) Segmentation of slice $S$. (e) Global labeling of slice $I$ by propagating segmentation from (d). Note that the center grain in (d) disappears in slice $I$, as desired.

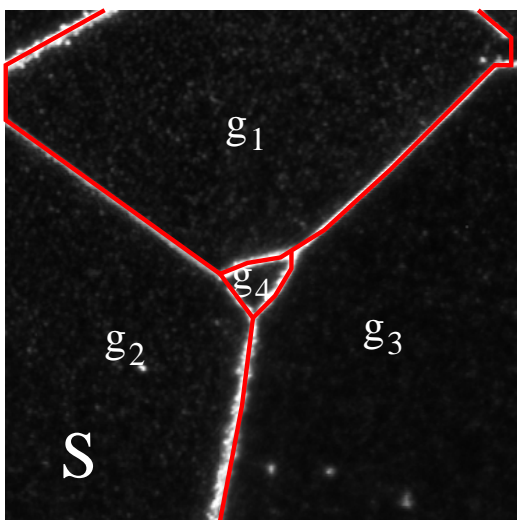

(a)

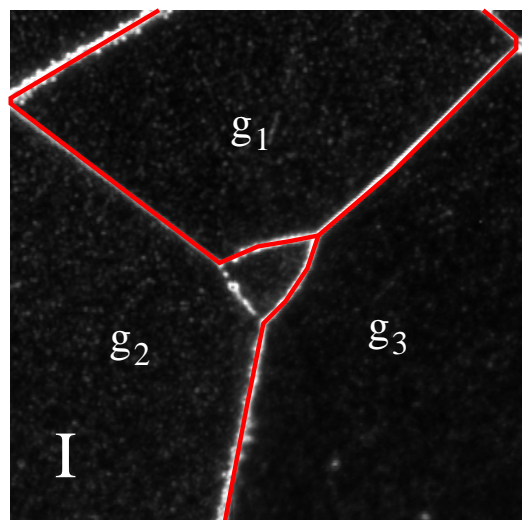

(b)

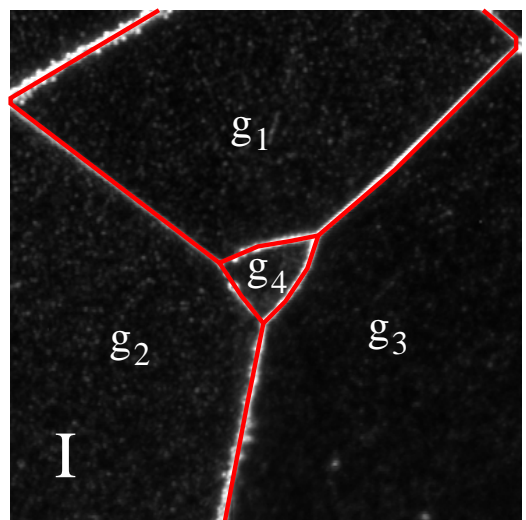

(c)

Figure 6: An illustration of Problem II. (a) Slice $S$, where $g_{4}$ is labeled. (b) Slice $I$, where $g_{4}$ is undesirably missed in global labeling. (c) The local relabeling result on slice $I$ after applying Strategy II. 


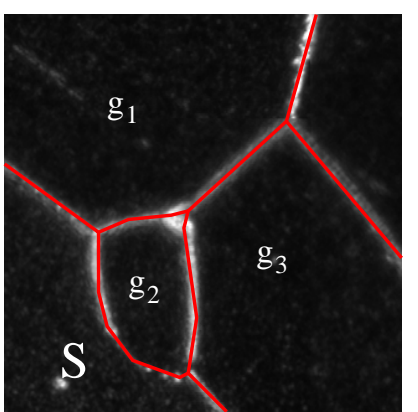

(a)

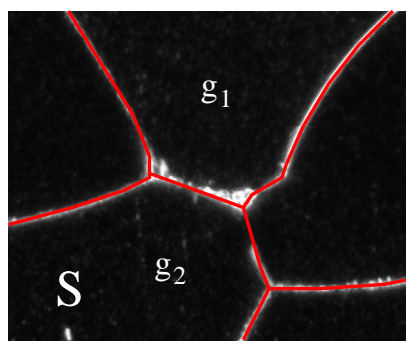

(d)

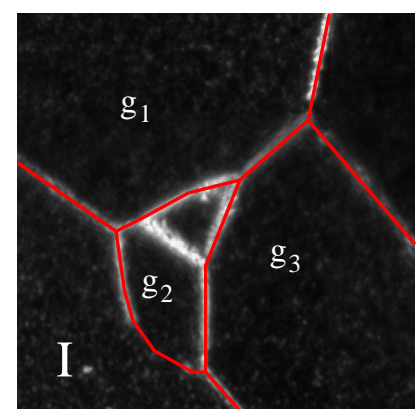

(b)

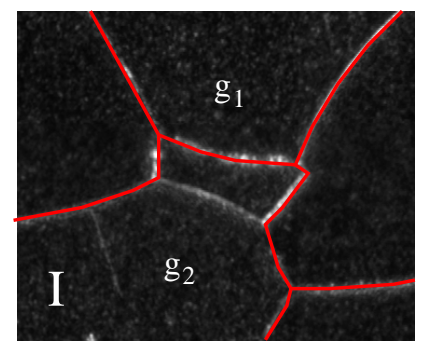

(e)

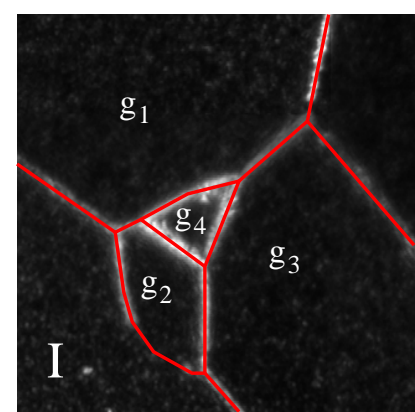

(c)

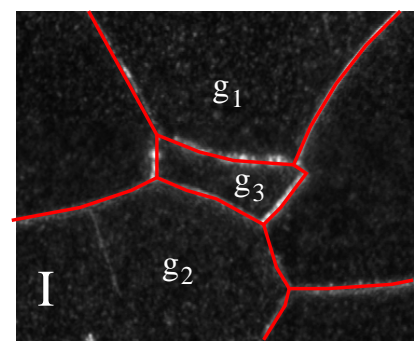

(f)

Figure 7: An illustration of Problem III. (a) Slice $S$, where $g_{4}$ is not present. (b) Slice $I$ with a new grain $g_{4}$ but not detected by the global labeling. (c) The local relabeling result on slice $I$ after applying Strategy II. (d-f) Another example analogue to the one shown in (a-c). The difference is that the appearance of $g_{4}$ changes the pairwise neighboring relations of other grains defined in $S$ and the local relabeling result in (f) is achieved using Strategy III.

Strategy I: For each small segment $g$ resulting from the global labeling, we evaluate its grain likeliness, which is based on $\bar{I}(g)$, the average intensity along the boundary of $g$ in slice $I$. If $\bar{I}(g)$ is low by a given criterion, we consider it to be a potentially undesirable grain as described in Problem I. We identify a local region which consists of grain $g$ and all its neighboring grains from the global labeling, as shown in Fig. 8 (a). In this local region, we rerun the labeling by using the data term defined in the global labeling and the smoothness term updated by removing the infinity cost in Eq. (2) for non-neighboring grains. This way, it is possible to remove $g$ as desired, since the optimal labeling algorithm favors the usage of fewer labels. If $g$ is indeed removed, we update the global labeling result by incorporating the changes made in this local relabeling. One example of applying this strategy to address Problem I is shown in Fig. 5 (c). The criteria for evaluating the grain likeliness will be discussed in Section 5 .

Strategy II: We treat each "Y" junction $P$ found in the global labeling on slice $I$ as a potential location of Problem II or Case I of Problem III. We identify a local region that consists of its three neighbors $g_{1}, g_{2}$, and $g_{3}$, as shown in Fig. 8 (b) and rerun the optimal labeling in this local region. For the data term and smoothness term in this local region, we make the following updates. First, we construct a small circular region $R$ around $P$, with radius $r$ as the zero data-term region for a new label $g_{R}$, as shown in Fig. 8(b). As before, the smoothness term is updated by removing the infinity cost in Eq. (2). The zero data-term regions for $g_{1}, g_{2}$, and $g_{3}$ are the same as the global labeling. In addition, we select seeds for $g_{1}, g_{2}, g_{3}$, and $g_{R}$ to prevent the removal of any of these four labels in the local relabeling. For a seed pixel associated with a label, we define the data term to be infinity cost when this pixel is assigned any other label.

As shown in Fig. 8 (b), we use the centroids of $g_{1}, g_{2}$, and $g_{3}$ as their respective seeds. We then uniformly sample points (e.g., $c_{1}, c_{2}, c_{3}$ in Fig. 8(b)) along a circle around $P$ with a radius $\frac{r}{2}$ as candidate seeds of $g_{R}$. With each candidate seed $c_{i}$ of $g_{R}$, together with the seeds for $g_{1}, g_{2}$, and $g_{3}$, we run the optimal labeling in this local region, attemping to find grains that were undesirably missed in Problem II and Case I of Problem III. Specifically, for each candidate seed of $g_{R}$, this local relabeling is used to generate a new segment $g_{R}$, for which we calculate its grain likeliness as defined in Strategy I. We take the local relabeling from the candidate seed 


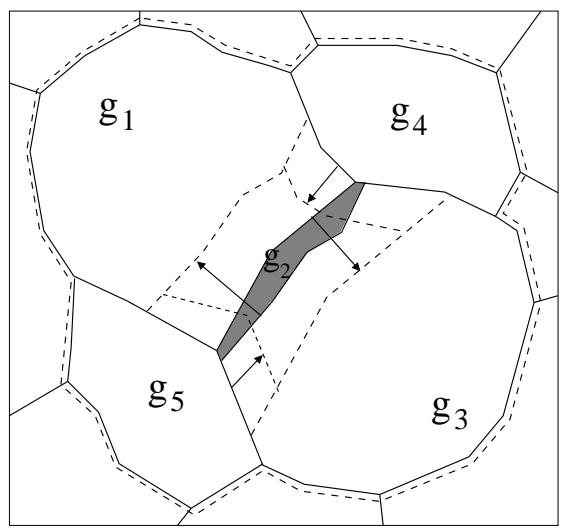

(a)

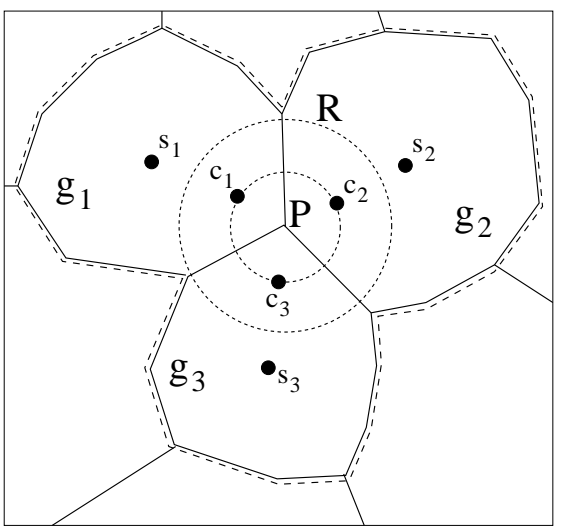

(b)

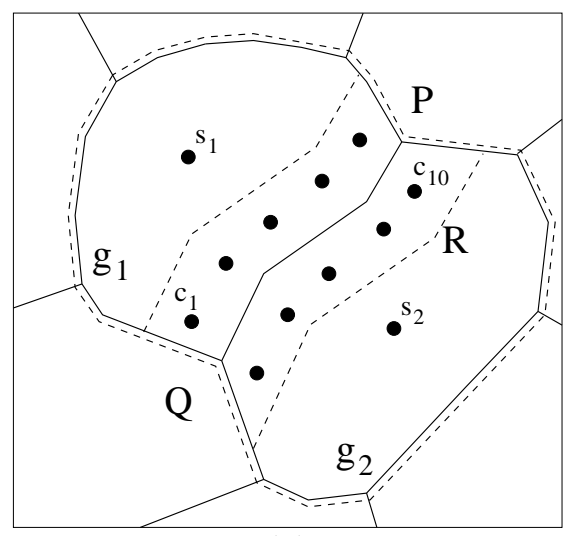

(c)

Figure 8: Illustration of Strategies I, II, and III. (a) Remove undesired grain $g_{2}$ using Strategy I. Arrows indicate the dilation direction of grain $g_{1}, g_{2}, g_{3}$ and $g_{4}$. Interior dashed curves indicate the dilated boundary of these four grains. (b) Finding new or mistakenly removed grains around a "Y" junction $P$ using Strategy II. (c) Finding new grains around a boundary fragment $P Q$ using Strategy III.

that results in a new grain $g_{R}$ with the largest grain likeliness. If this grain likeliness is high, we update the global labeling result by incorporating the changes made in this local relabeling. An example of applying this strategy to address Problem II is shown in Fig. 6 (c), and an example of applying this strategy to address Case I of Problem III is shown in Fig. 7 (c).

Strategy III: We treat each boundary fragment $P Q$ shared by two grains $g_{1}$ and $g_{2}$ found in the global labeling on slice $I$ as a potential location for Case II of Problem III. We identify a local region that consists of $g_{1}$ and $g_{2}$, as shown in Fig. 8 (c) and rerun the optimal labeling in this local region. For the data term in this local region, we construct a region $R$ by dilating the fragment $P Q$ and use this region as the zero data-term region for a new label $g_{R}$, as shown in Fig. 8 (c). The smoothness term is, as before, updated by removing the infinity cost in Eq. (2). As shown in Fig. 8 (c), we use the centroids of $g_{1}$ and $g_{2}$ as their respective seeds. We then uniformly sample points (e.g., $c_{1}, \ldots, c_{10}$ in Fig. 8 (c)) in $R$ along both sides of $P Q$ as candidate seeds of $g_{R}$. The distance between each candidate and the fragment $P Q$ is set to be half of the dilation size in constructing $R$. Similar to Strategy II, we run optimal labeling for each candidate seed to identify new potential grains as described in Case II of Problem III and update the global labeling result when necessary. An example of applying this strategy to address Case II of Problem III is shown in Fig. 7 (f).

\section{EXPERIMENTS}

To evaluate the performance of the proposed method, we collect a sequence of 11 microscopic titanium images. We start with the ground-truth segmentation on the first slice and propagate it to segment the second slice. We then propagate the segmentation on the second slice to segment the third slice. This process is repeated ten times to segment all slices. The proposed method, which combines a global labeling and multiple local relabelings, is used for each step of the propagation. Each image slice has a resolution of $750 \times 525$. The dilation size is set to 20 pixels for constructing the zero data-term region in the global labeling. The radius of the region $R$ is set to 30 pixels in Strategy II, and the dilation size for region $R$ is set to 40 pixels in Strategy III. These values are selected according to the intra-slice resolution. In our experiments, grain likeliness is considered to be high if $66 \%$ of the pixels along its boundary have an intensity that is larger than $\frac{3}{2} \bar{I}$, where $\bar{I}$ is the average intensity of all the pixels in slice $I$. Otherwise, we consider this grain likeliness to be low. This makes the grain likeliness evaluation adaptive to the overall brightness of a considered slice. In Strategy I, we consider a grain to be small if it contains less than 200 pixels, and we proceed to run local relabeling for potential removal of this grain.

For performance evaluation, we have manual ground-truth segmentations of all 11 slices provided by materials scientists. The grain boundaries in the ground truth are roughly five pixels wide, as shown in Fig. 10. We evaluate a segmentation result by computing the coincidence between the detected boundaries and the ground 


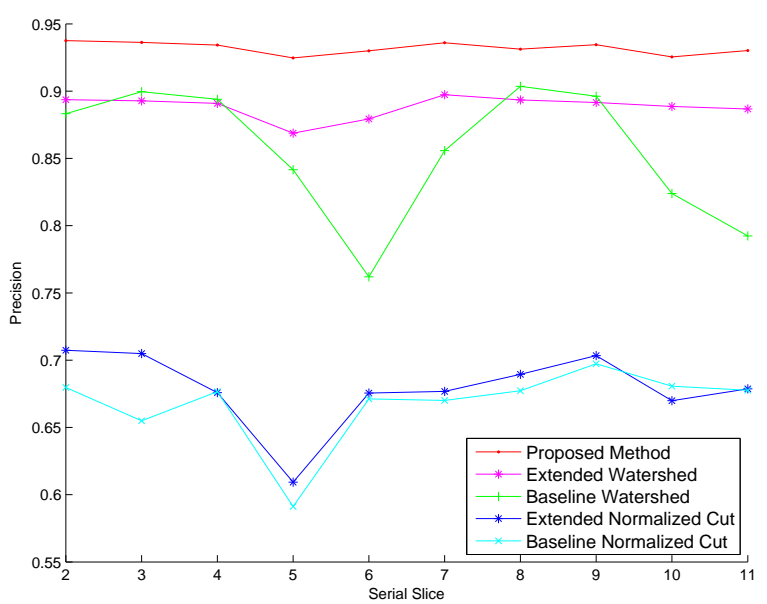

(a) Precision

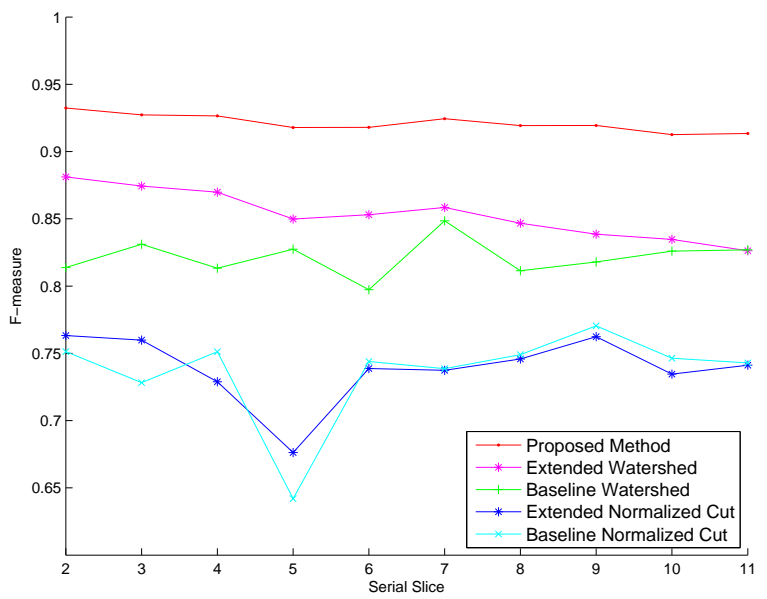

(c) F-measure

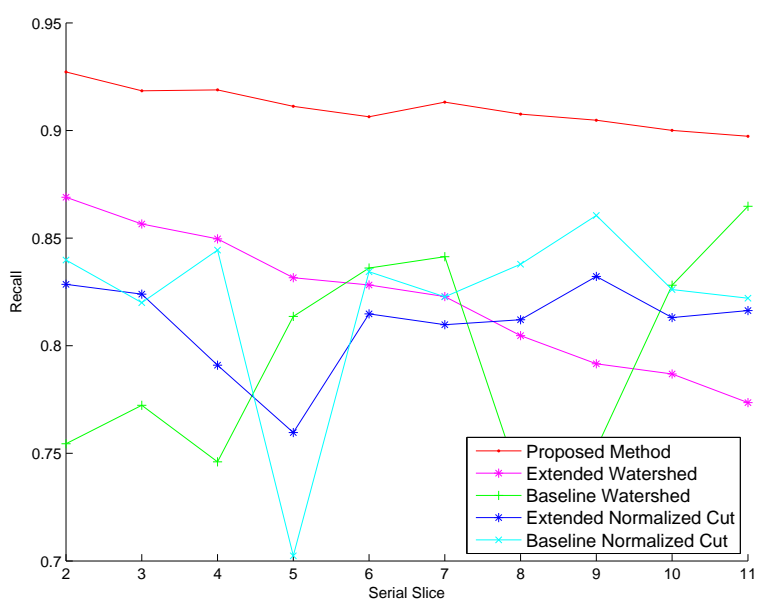

(b) Recall

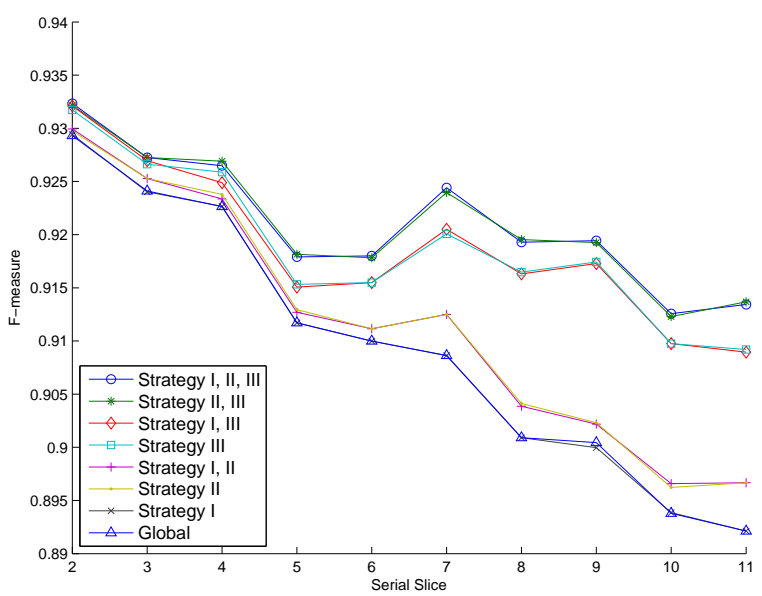

(d) Strategies

Figure 9: (a-c) The segmentation precision, recall, and F-measures for the proposed method, the watershed method, and normalized cut on the 10 tested slices. (d) Performance curves on the 10 tested titanium slices using subsets of the three strategies. The curve labeled "Global" represents the performance using only the global labeling. Note that the scale for (d) is different from (a-c).

truth boundaries. We dilate the detected boundary to a width of five pixels and then calculate the precision and recall on 10 slices, leaving out the first slice, where the ground-truth segmentation is used as the initialization of propagation. We also calculate a summary F-measure that integrates the precision and recall. ${ }^{25}$

For comparison, we run the watershed method, ${ }^{10}$ using a Matlab implementation based on,${ }^{26}$ and the normalized cut method, ${ }^{13}$ using a linear-time multiscale implementation based on, ${ }^{27}$ on the same 10 image slices. In addition, we create two extensions of both of these methods so that they operate more appropriately on metallic image slices. We test two settings for each of these methods: the case of dilating their detected boundaries to a width of five pixels before measuring their correlation with the ground truth, and the case of no dilation, selecting the case that leads to a better F-measure.

For the unmodified watershed method, which we call the baseline watershed method, we tested 50 different minima suppression levels and selected the value that leads to the best F-measure. Furthermore, similar to, ${ }^{15}$ we create an extended watershed implementation using markers to incorporate the same ground truth segmentation used by the proposed method, and propagate these markers in the exact same manner as the proposed method 
for a completely fair comparison using the exact same initial segmentation.

The unmodified normalized cut implementation, which we call the baseline normalized cut, requires the number of segments as input, so we provide the exact number of segments from the ground truth for each slice. In addition, since the high intensity regions in the metallic images - rather than the gradient of the imagecorrespond to the grain boundaries, we create an extended normalized cut that computes its graph edge weight directly from the image intensity rather than the gradient of the image intensity.

The segmentation precision, recall, and F-measures for all methods on the 10 tested slices are shown in Fig. $9(\mathrm{a}-\mathrm{c})$. Overall, the proposed method scores higher than $90 \%$ for both precision and recall, even after propagating to a slice that is 10 slices away from the initial slice, and shows much better performance than the extended and baseline versions of watershed and normalized cut.

Although the baseline watershed method has been used to segment images with many grains or cells,${ }^{12}$ its performance on this problem is not satisfactory, producing relatively high precision and low recall on many slices. This indicates that such high precision is due to oversegmentation. The extended watershed method performs better than the baseline watershed method. However, even with the propagation afforded by the extended watershed method, the recall consistently drops, as extended watershed is not capable of identifying new grains as they appear when the series section plane moves to a new slice. This is evident by the many missed grain boundary segments in the last image of the second row of Fig. 10. As a state-of-the-art image segmentation method, normalized cut produces low precision and recall. This is partially due to normalized cut placing boundaries through grain centers because of scratches and other noise present inside the grains. The extended normalized cut has similar performance to the baseline version.

Figure 9 (d) shows the improvements gained by each of the three local relabeling strategies. We can see that, overall, the inclusion of each strategy increases the segmentation performance. From this, Strategy I does not have a large impact on the performance. This is due to the fact that this strategy acts only on very small grains and its contribution to overall precision and recall is low. However, the identification of all the grains, either small or large, while maintaining consistent topology is nonetheless important in many material applications, which may not be well reflected by these overall precision and recall metrics. Thus, the small change made in Strategy I may be important in some material applications. Strategy III introduces the largest improvement among all three local-labeling strategies. This is because there are many instances of Case II of Problem III, which may substantially affect the segmentation performance when this segmentation is propagated to the next slice.

We conducted all our experiments on $2 \mathrm{GHz}$ Linux workstations with $8 \mathrm{~GB}$ of memory. Each propagation requires roughly 15 minutes to complete when running both global labeling and local relabeling. Without local relabeling, the propagation completes in roughly 5 minutes. In all cases, the run time is dominated by the graphcut computation - all other computations we performed introduce negligible overhead (i.e., less than 15 seconds).

Figure 10 shows the segmentation results on selected slices using the proposed method, the watershed method, and normalized cut, along with the ground truth. We only present the better of the baseline or extended version of each method. We can see that the proposed method can better detect indistinct grain boundaries and is immune to the strong, high-intensity scratch lines, while the extended watershed method is unable to handle inconsistency, and normalized cut suffers in the presence of strong noise.

\section{CONCLUSION}

In this paper, we developed an optimal labeling-based approach for image sequence segmentation and illustrated its effectiveness on a series of metallic image slices. In propagating the 2D segmentation from one slice to another, we first ran a global labeling to enforce consistent 2D structure topology. We then used a local relabeling process to identify and correct possible topology inconsistency to better segment multiple contiguous substructures within a slice. We tested the proposed method on a sequence of serial-section slices of a titanium sample and achieved promising performance that is superior to the well-known watershed and normalized cut methods.

Acknowledgements: We would like to thank Dave Rowenhorst from the Navy Research Lab for providing the titanium serial section images. 

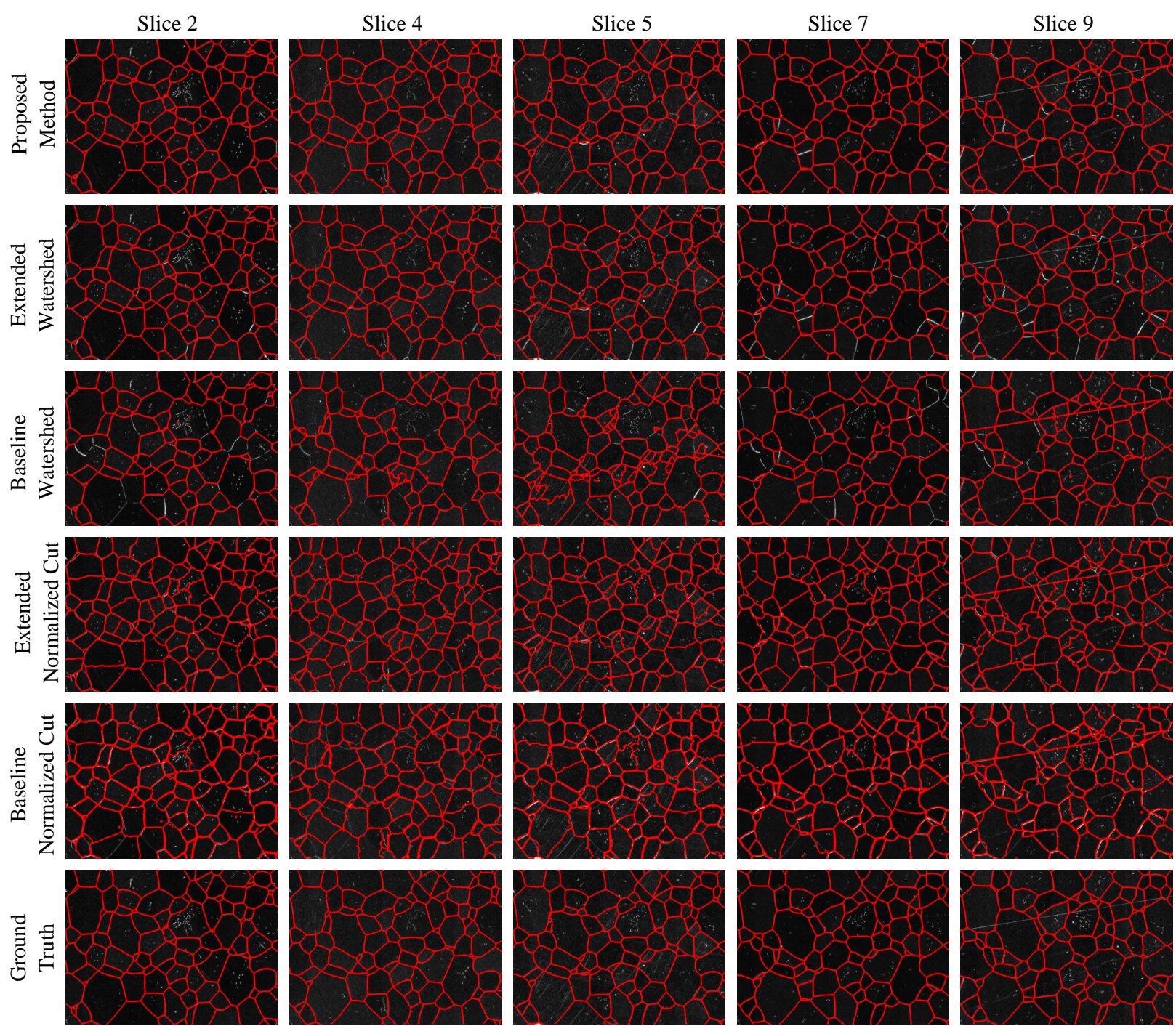

Figure 10: Segmentation results on selected slices using the proposed method, watershed, and normalized cut, along with the ground truth.

This work was funded, in part, by AFOSR FA9550-11-1-0327, NSF-1017199, NSF-0951754, and Army Research Laboratory (ARL) under Cooperative Agreement Number W911NF-10-2-0060. The views and conclusions contained in this document are those of the authors and should not be interpreted as representing the official policies, either express or implied, of AFOSR, NSF, ARL or the U.S. Government. The U.S. Government is authorized to reproduce and distribute reprints for Government purposes, notwithstanding any copyright notation herein.

\section{REFERENCES}

[1] Khan, S. and Shah, M., "Object based segmentation of video using color, motion and spatial information," in [IEEE Conference on Computer Vision and Pattern Recognition], II-746-II-751 vol.2 (2001).

[2] Fu, K. and Mui, J., "A survey on image segmentation," Pattern Recognition 13(1), 3 - 16 (1981).

[3] Brahme, A., Alvi, M., Saylor, D., Fridy, J., and Rollett, A. D., "3D reconstruction of microstructure in a commercial purity aluminum," Scripta Materialia 55(1), 75-80 (2006).

[4] Szeliski, R., Tonnesen, D., and Terzopoulos, D., "Modeling surfaces of arbitrary topology with dynamic particles," in [IEEE Conference on Computer Vision and Pattern Recognition], 140-152 (June 1993). 
[5] Mcinemey, T. and Terzopoulos, D., "Topology adaptive deformable surfaces for medical image volume segmentation," Medical Imaging, IEEE Transactions on 18(10), 840-850 (1999).

[6] Yi, J. and Ra, J. B., "A locally adaptive region growing algorithm for vascular segmentation," Imaging Systems and Technology 13(4), 208-214 (2003).

[7] Zhou, J., Lim, T., Chong, V., and Huang, J., "Segmentation and visualization of nasopharyngeal carcinoma using MRI," Computers in Biology and Medicine 33(5), 407-424 (2003).

[8] Shi, J. and Malik, J., "Motion segmentation and tracking using normalized cuts," in [IEEE International Conference on Computer Vision], 1154-1160 (2008).

[9] Rueckert, D. and Burger, P., "Geometrically deformable templates for shape-based segmentation and tracking in cardiac MR image," in [International Workshop on Energy Minimization Methods in CVPR], 83-98 (1997).

[10] Vincent, L. and Soille, P., "Watersheds in digital spaces: An efficient algorithm based on immersion simulations," IEEE Transactions on Pattern Analysis and Machine Intelligence 13(6), 583-598 (1991).

[11] Mangan, A. P. and Whitaker, R. T., "Partitioning 3D surface meshes using watershed segmentation," IEEE Transactions on Visualization and Computer Graphics 5, 308-321 (October 1999).

[12] Li, Q., Ni, X., and Liu, G., "Ceramic image processing using the second curvelet transform and watershed algorithm," in [IEEE International Conference on Robotics and Biomimetics], 2037-2042 (2007).

[13] Shi, J. and Malik, J., "Normalized cuts and image segmentation," IEEE Transactions on Pattern Analysis and Machine Intelligence 22(8), 888-905 (2000).

[14] Gamio, J. C., Belongie, S., and Majumdar, S., "Normalized cuts in 3D for spinal MRI segmentation," IEEE Transactions on Pattern Analysis and Machine Intelligence 26(1), 36-44 (2004).

[15] Chae, Y.-s. and Kim, D., "Automatic marker-driven three dimensional watershed transform for tumor volume measurement," in [International Conference on Advances in Hybrid Information Technology], 149158 (2007).

[16] Li, D., Rollett, A. D., Vialle, G., and Garmestani, H., "Multiproperty microstructure and property design of magnetic materials," Journal of Engineering Materials and Technology 130(2), 21-23 (2008).

[17] Canny, J., "A computational approach to edge detection," IEEE Transactions on Pattern Analysis and Machine Intelligence 8(6), 679-698 (1986).

[18] Maire, M., Arbelaez, P., Fowlkes, C., and Malik, J., "Using contours to detect and localize junctions in natural images," in [IEEE Conference on Computer Vision and Pattern Recognition], 1-8 (2008).

[19] Sezgin, M. and Sankur, B., "Survey over image thresholding techniques and quantitative performance evaluation," Journal of Electronic Imaging 13(1), 146-165 (2004).

[20] Museth, K., Breen, D. E., Zhukov, L., and Whitaker, R. T., "Level set segmentation from multiple nonuniform volume datasets," in [IEEE Visualization], 179-186 (2002).

[21] Boykov, Y., Veksler, O., and Zabih, R., "Fast approximate energy minimization via graph cuts," IEEE Transactions on Pattern Analysis and Machine Intelligence 23(11), 1222-1239 (2001).

[22] Boykov, Y. and Kolmogorov, V., "An experimental comparison of min-cut/max- flow algorithms for energy minimization in vision," IEEE Transactions on Pattern Analysis and Machine Intelligence 26(9), 1124-1137 (2004).

[23] Kolmogorov, V. and Zabin, R., "What energy functions can be minimized via graph cuts?," IEEE Transactions on Pattern Analysis and Machine Intelligence 26(2), 147-159 (2004).

[24] Veksler, O., Efficient graph-based energy minimization methods in computer vision, PhD thesis, Cornell University, Ithaca, NY, USA (1999).

[25] Martin, D., Fowlkes, C., Tal, D., and Malik, J., "A database of human segmented natural images and its application to evaluating segmentation algorithms and measuring ecological statistics," in [IEEE International Conference on Computer Vision], 2, 416-423 (2001).

[26] Meyer, F., "Topographic distance and watershed lines," Signal Processing 38(1), 113-125 (1994).

[27] Cour, T., Benezit, F., and Shi, J., "Spectral segmentation with multiscale graph decomposition," in [IEEE Conference on Computer Vision and Pattern Recognition], 1124-1131 (2005). 